\title{
Surgical Excision of Dumbbell-shaped Vagus Nerve Schwannoma: A Case Report
}

Amy O., Kalai T., Anusha B., Zakinah Y

Department of Otorhinolaryngology, Hospital Kuala Lumpur

\section{ABSTRACT}

Schwannoma is a benign peripheral nerve sheath tumour that arises from the uncontrolled proliferation of Schwann cells. Intracranial and extracranial vagus nerve schwannoma is an extremely rare entity that poses a surgical challenge due to its delicate location in the cerebrum and parapharyngeal space adjacent to major neck vessels. This report describes a unique case of a 42 years old female with left dumbbell vagus nerve schwannoma, involving both the intracranial and extracranial portion of the left vagus nerve, extending into the parapharyngeal space. She underwent a two-stage surgery in which intracranial vagus schwanomma was debulked initially, and excision of the extracranial schwanomma in the parapharyngeal space was performed in a later setting via a transcervical approach.

KEYWORDS: Vagus nerve, Vagal Schwannoma, Dumbbell Tumour

\section{INTRODUCTION}

Schwannoma is a tumour caused by uncontrolled proliferation of Schwann cells located in the myelin sheath. It is a benign, well-encapsulated and slowgrowing nerve sheath tumour that grows at a rate of $3.2 \mathrm{~mm} /$ year. ${ }^{1}$ It was reported that up to $45 \%$ of the extracranial schwannoma occurs in the head and neck region. ${ }^{2}$ Clinically, intracranial schwannoma presents with headache, cranial nerve palsies specific to the cranial nerve involved, and in severe cases, signs of raised intracranial pressure if left untreated. On the other hand, an extracranial vagal schwannoma of the neck presents with progressively growing painless neck swelling commonly associated with hoarseness and obstructive symptoms. The standard management of benign schwannomas differs greatly according to the size of the tumour, the presentation of symptoms, the local surgical expertise, and the patient's wishes. Complete surgical excision with neural preservation remains the gold standard treatment for symptomatic benign schwannoma. ${ }^{3}$

Corresponding Author:

Dr. Amy Oon Li Ling

Department of Otorhinolaryngology,

Hospital Kuala Lumpur,

23 Jalan Pahang, 53300 Kuala Lumpur,

Wilayah Persekutuan, Malaysia

Tel No : +60164452289

Email : amylingoon@gmail.com

\section{CASE REPORT}

A 42 year old woman was referred for left-sided neck swelling. She was under neurosurgical follow up since 2017 for recurrent headaches during which magnetic resonance imaging (MRI) of the brain revealed an intracranial space-occupying lesion over the jugular foramen. The patient underwent debulking of the tumour via left retrosigmoid craniotomy two years ago, during which the histopathology confirmed the diagnosis of schwannoma. A full excision of the tumour was not achieved, due to its proximity to cranial nerves $X$, $\mathrm{XI}$, and XII. Postoperatively, she had left cranial nerve $\mathrm{X}, \mathrm{XI}$ and XII palsy.

She presented with complaints of dysphagia, intermittent shortness of breath which worsened upon lying down, hoarseness and gradual increase of neck swelling for one year since her initial visit. She had no constitutional symptoms. A physical examination revealed a left sided level II and III neck swelling, extending from skull base to the hyoid bone, measuring $7 \mathrm{~cm} \times 10 \mathrm{~cm}$. It was soft, smooth, fixed, non-tender, well-circumscribed and non-pulsatile. The overlying skin was in normal condition. Flexible nasoendoscopy demonstrated medialization of the left oropharynx with left vocal cord abductor palsy in the paramedian position, which was well compensated by the right vocal cord.

A brain and neck MRI showed a T2 enhancing mass 
with cystic component medial to the left carotid sheath, measuring $6.7 \times 2.4 \times 10.9 \mathrm{~cm}$, causing a mass effect on the nasopharynx and oropharynx. The mass extended into the left jugular foramen, with widening of the canal. Intracranially, it extended into the left cerebellomedullary extradural space. A fine needle aspiration and cytology (FNAC) report came back as schwannoma.

Transcervical excision of the tumour was planned for this patient, due to distressful obstructive symptoms. Neurosurgical opted for radiotherapy intervention against surgical management because of the delicacy of the tumour location, size and the high risk of complications.

Prior to surgery, she underwent a preoperative diagnostic angiogram, as there was a potential need for carotid artery ligation. Under general anaesthesia, a transcervical incision was made and dissected along the planes until the carotid sheath was breached. Intraoperatively, a grossly ovoid wellencapsulated mass was seen splaying the left internal carotid artery and the internal jugular vein. The tumour was densely adhered to the left vagus nerve, which was sacrificed [Fig. 1]. The left common carotid artery, left internal carotid artery, left external carotid artery, internal jugular vein, cervical sympathetic chain and spinal accessory nerve were preserved [Fig.2]. The excised tumour is shown in Figure 3. Postoperatively, the patient developed rebound tachycardia, which was resolved with the commencement of a rate-limiting betablocker drug Bisoprolol. She was subsequently discharged home well on postoperative day 11 .

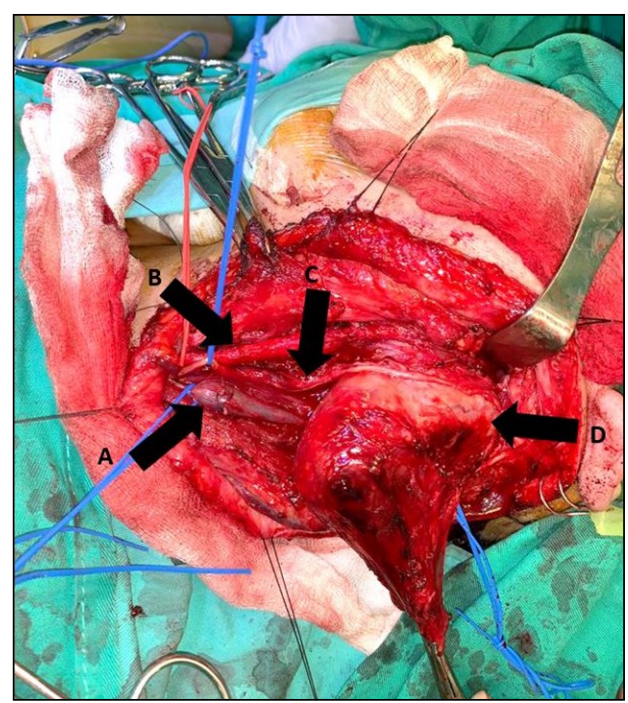

Figure 1: Clinical picture showing the position of the tumour in relation to neck structures. A: left internal jugular vein, B: left common carotid artery, C: left vagus nerve adhering to the tumour and $D$ : tumour.

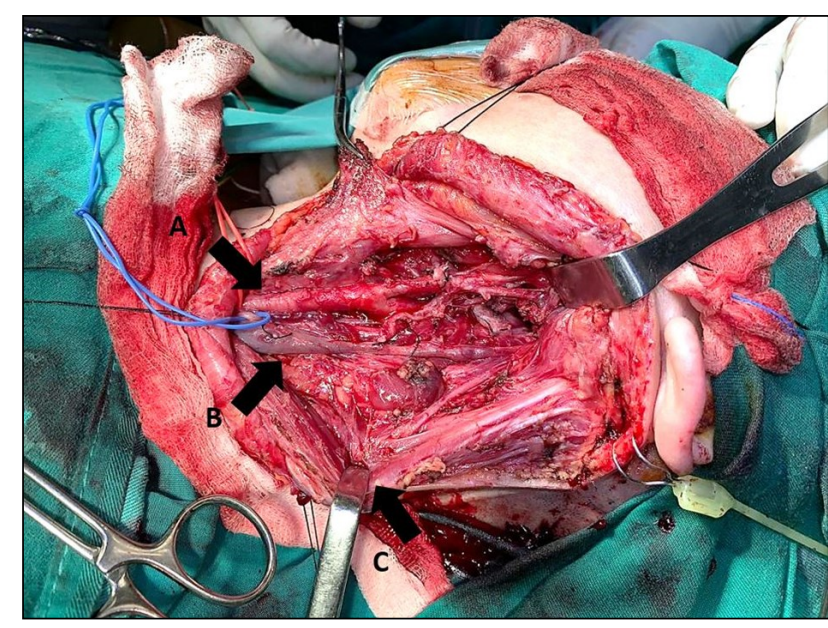

Figure 2: Clinical picture showing the tumour bed after tumour excision. A: left common carotid artery, B: left internal jugular vein and $\mathrm{C}$ : left retracted sternocleidomastoid muscle.

\section{DISCUSSION}

Schwanomma is a peripheral nerve sheath tumour that is most commonly benign, but malignant transformation has been reported. ${ }^{4}$ Most commonly intracranial, some schwannomas have the tendency to grow to a large size that extends into the parapharyngeal space. Vagal schwannomas are seen in the third and fifth decades of life, but do not show any sex or racial predilection. ${ }^{5}$ Clinically, they range from being asymptomatic to a wide range of symptoms, including but not limited to headache, seizures, raised intracranial pressure, neck swelling, hoarseness and dysphagia. The presence of a paroxysmal cough upon palpation of neck mass is suggestive of cervical vagal schwannoma.

Lateral neck masses have a myriad of differential diagnoses, and this poses a diagnostic dilemma. Differentials of lateral neck masses include branchial cleft cyst, benign or malignant lymphadenopathy, carotid body tumour and

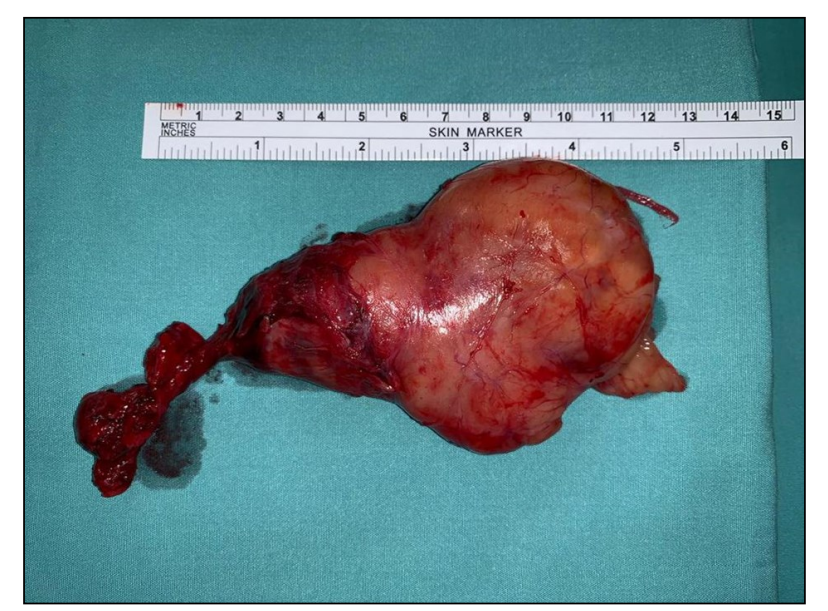

Figure 3: The excised well-encapsulated tumour measuring $13 \mathrm{~cm} \times 6 \mathrm{~cm}$ 


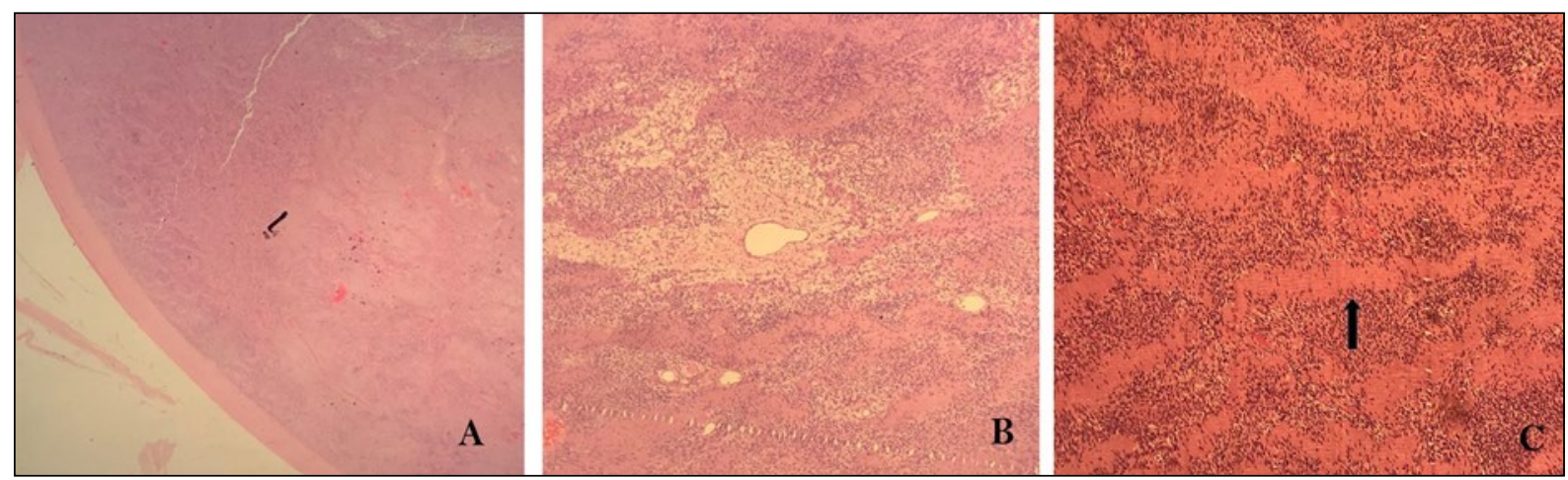

Figure 4: The histological slides of the tumour. A: well-encapsulated tumour, B: the presence of Antoni A and Antoni B hypercellular and hypocellular cells, and C: the presence of Verocay bodies as pointed by the arrow.

paraganglioma. Fine needle aspiration cytology (FNAC) is controversial, and prior research has shown that diagnosing schwannoma using FNAC could be difficult, and its accuracy has been reported to be only around $20 \%{ }^{6}$ Under the microscope, schwannoma is a biphasic tumour consisting of hypercellular Antoni A cells intertwining with hypocellular Antoni B cells with Verocay bodies and a hyalinized vessel wall [Fig. 4]. A benign schwannoma does not show any nuclear atypia or high mitotic index. ${ }^{7}$

In addition to FNAC, we employed a MRI as the investigation of choice in aiding our diagnosis of vagal schwannoma. ${ }^{8,9}$ Yasumatsu et al. reported $80 \%$ diagnosis of schwannoma by using a MRI. ${ }^{6}$ It allows superior soft-tissue visualization that allows us to identify the exact site, extent and size of the tumour, and most importantly distinguish between a vagal schwannoma and a tumour from the cervical sympathetic chain. ${ }^{9}$ A vagal schwannoma typically splays the internal jugular vein and the internal carotid artery, whereas a cervical sympathetic chain schwannoma does not cause separation of any major neck vessels. ${ }^{9}$ Furthermore, a multi-planner thinsliced MRI scan provides a detailed guide in anatomy, and enhances preoperative surgical planning that is essential in view of the delicate anatomy of the neck.

Suarez et al. suggested an angiogram as a complementary investigation for a parapharyngeal tumour. ${ }^{10}$ We proceeded for a diagnostic angiogram; this is for a detailed mapping of the carotid vessels and its collaterals, should the need to ligate the internal carotid artery arise intraoperatively. Fortunately, the angiogram showed good anterior and posterior communications, and minor tumour feeder vessels that did not require embolization.

The gold standard for treatment is a complete surgical excision of the tumour with neural preservation. ${ }^{6}$ To prevent the risk of nerve injury, an intraoperative nerve monitoring system must be employed with electromyographic intubation. Surgical excision is only done when highly indicated, and this includes symptomatic patients, suspicion of malignancy and cosmetic purposes as per the patient's request. We highly suggest conservative watchful treatment with serial imaging every six months for asymptomatic tumours.

Our patient underwent a two-stage surgery craniotomy and retrosigmoid approach with tumour debulking by neurosurgical, followed by excision of left vagus nerve schwannoma via transcervical approach, two years later. Both surgeries were planned solely based on the debilitating symptoms that the patient experienced. Depending on the length excised, anastomosis can be done end to end or transposition with the great auricular nerve. We can almost always expect vocal cord palsy postoperatively. Aggressive speech and swallowing rehabilitation should be started as soon as possible to prevent the risk of aspiration. Based on our experience, the excision of the cervical vagus nerve results in postoperative tachycardia due to lack of rate inhibition that the vagus nerve previously provided. Therefore, the patient had to be started on a lifelong rate-limiting beta-blocker Bisoprolol. In conclusion, it is extremely vital to weigh the pros and cons of excising an intracranial and extracranial benign schwannoma, although the presence of schwannoma may impair the patient's quality of life; the surgical excision of it poses other potential complications that could significantly affect the 
patient's daily life. The patient's expectations and concerns must be clarified before making the decision to remove the tumour.

\section{REFERENCES}

1. Charabi S, Thomsen J, Mantoni M, Charabi B, Bjarne JR, Sven Erik BR, Gyldensted C, Tos M. Acoustic neuroma (vestibular schwannoma): growth and surgical and nonsurgical consequences of the wait-and-see policy. Otolaryngology-Head and Neck Surgery. 1995 Jul;113(1):5-14.

2. Colreavy MP, Lacy PD, Hughes J, BouchierHayes D, Brennan P, O'Dwyer AJ, Donnelly MJ, Gaffney R, Maguire A, O'Dwyer TP, Timon CV. Head and neck schwannomas-a 10 year review. The Journal of Laryngology \& Otology. 2000 Feb;114(2):119-24.

3. Kang GC, Soo KC, Lim DT. Extracranial nonvestibular head and neck schwannomas: a tenyear experience. ANNALS-ACADEMY OF MEDICINE SINGAPORE. 2007 Apr 1;36(4):233.

4. Li C, Chen Y, Zhang H, Zheng X, Wang J. Epithelioid angiosarcoma arising in schwannoma: report of three Chinese cases with review of the literature. Pathology international. $2012 \mathrm{Jul} ; 62(7): 500-5$.

5. Chiofalo MG, Longo F, Marone U, Franco R, Petrillo A, Pezzullo L. Cervical vagal schwannoma. A case report. Acta Otorhinolaryngologica Italica. 2009 Feb;29 (1):33.

6. Yasumatsu R, Nakashima T, Miyazaki R, Segawa $Y$, Komune $S$. Diagnosis and management of extracranial head and neck schwannomas: a review of 27 cases. International journal of otolaryngology. 2013 May 8;2013.

7. Dahl I, Hagmar B, Idvall I. Benign Solitary Neurilemoma (Schwannoma) A Correlative Cytological and Histological Study of 28 Casesd. Acta Pathologica Microbiologica Scandinavica Series A: Pathology. 1984 Mar;92(1-6):91-101.

8. Kami YN, Chikui T, Okamura K, Kubota $Y$, Oobu K, Yabuuchi H, Nakayama E, Hashimoto K, Yoshiura $\mathrm{K}$. Imaging findings of neurogenic tumours in the head and neck region. Dentomaxillofacial Radiology. 2012 Jan;41 (1):18-23.

9. Furukawa M, Furukawa MK, Katoh K, Tsukuda M. Differentiation between schwannoma of the vagus nerve and schwannoma of the cervical sympathetic chain by imaging diagnosis. The Laryngoscope. 1996 Dec;106(12):1548-52.

10. Suárez-Fente V, Llorente-Pendás JL, GómezMartínez J, García-González LA, López-Álvarez F, Suárez-Nieto C. Primary tumours of the parapharyngeal space. Our experience in 51 patients. Acta Otorrinolaringologica (English Edition). 2009 Jan 1;60(1):19-24. 